\title{
Decreasing Lung Cancer Mortality through Screening-The Lung Cancer Alliance Experience
}

\author{
Laurie Fenton Ambrose, Amy Copeland, and Jennifer C King
}

Lung Cancer Alliance, Washington, DC, US

DOI: https://doi.org/10.17925/OHR.2017.13.01.17

ver the past several years, detection of lung cancer has changed dramatically due to critical scientific advances, adapting guidelines, and key policy and coverage decisions. Lung Cancer Alliance provides an example of how patient advocacy organizations working alongside of scientists, clinicians, and policy-makers have been influential in shaping science and policy for the benefit of patients.

\section{Keywords}

Lung cancer, screening, imaging, patient advocacy

Disclosure: Laurie Fenton Ambrose, Jennifer C King, and Amy Copeland are affiliated with Lung Cancer Alliance. This article is a short opinion piece and has not been submitted to external peer reviewers. No funding was received in the publication of this article. Authorship: All named authors meet the International Committee of Medical Journal Editors (ICMJE) criteria for authorship of this manuscript, take responsibility for the integrity of the work as a whole, and have given final approval to the version to be published. open Access: This article is published under the Creative Commons Attribution Noncommercial License, which permits any noncommercial use, distribution, adaptation, and reproduction provided the original author(s) and source are given appropriate credit.

Received: March 6, 2017

Published Online: May 12, 2017

Citation: Oncology \& Hematology Review, 2017;13(1):17-8

Corresponding Author: Jennifer C King, PhD,

1700 K Street NW, Ste 660, Washington, DC

20006, US. E: jking@lungcanceralliance.org
Over the past several years, there has been a profound paradigm shift for those at high risk for lung cancer due to the development and release of scientific validation and national guidelines and recommendations for lung cancer screening. The lung cancer community is poised to realize an unprecedented scale and magnitude of benefit from early detection due to five-year survival rates of only $4.3 \%$ when lung cancer is diagnosed as metastatic disease versus $55.2 \%$ when confined to the primary site. ${ }^{1}$ Given the import and potential of this opportunity, there has been a clear need to develop programs and guidance to ensure the safe, responsible, and equitable implementation of lung cancer screening and to bring proper health messages to those at risk. Lung Cancer Alliance (LCA), a national non-profit cancer advocacy organization, recognized this gap and has stepped in to support the adoption of best practices and consumer safety measures, as well as public service messaging about screening risk and benefit.

Immediately upon the scientific validation of the mortality benefit of lung cancer screening from the National Lung Screening Trial (NLST), ${ }^{2}$ LCA moved rapidly to convene a multidisciplinary team of health care professionals to devise a blueprint to guide the responsible implementation of screening. The overarching goal embedded in this discussion was to ensure the public understood that they had a right to know they could be at risk for lung cancer and that they had a right to responsible care. And thus, the LCA National Framework for Excellence in Lung Cancer Screening and Continuum of Care was born in February 2012. ${ }^{3}$ Embedded within this national framework are the following guiding principles and elements:

- increasing the public's awareness about risk and their rights to responsible care, including educational resources and campaigns to spread this message;

- creating a framework of agreed upon best practices to guide the safe and responsible development of a screening Center of Excellence medical center network that then leveraged member resources to support each other; and

- creating collaborations and relationships with other professional and advocacy societies and outreach to public and private payers to effect change at the policy level.

There are currently more than 475 facilities within the LCA national network of Screening Centers of Excellence that have committed to following best practice principles of care. These practices include following approved protocols for screening and management of findings, appropriate communication of risks and benefits, linkage to a multidisciplinary care team, and referral to smoking cessation services. 
With the NLST to provide solid evidence from a large randomized controlled trial, LCA worked closely with a broad coalition of stakeholders to advocate for U.S. Preventive Services Task Force review and later to advocate for a coverage review and decision from Centers for Medicare and Medicaid Services (CMS). This alliance of stakeholders included the American College of Radiology and the Society of Thoracic Surgeons, and continues to monitor changes in coverage reimbursement and other policy concerns. Lung cancer screening is currently a covered benefit for most individuals at high risk, with no cost sharing, co-pays or deductibles. ${ }^{4}$

Although the mortality benefit of lung cancer screening was proven in the NLST, there remain many opportunities for further research to improve early detection of lung cancer. LCA is playing an active role, promoting research collaboration around implementation science and accurate risk prediction to ensure the proper population is screened. Since the NLST, clinical practice guidelines have continually been refined to reduce the number of false positive screens and unnecessary harm to the patient..$^{5-7}$ LCA has undertaken collaborative studies to understand and optimize practices within the network of Screening Centers of Excellence around many of the CMS-mandated services, such as smoking cessation and shared decision making. ${ }^{8}$ In addition, many researchers, including those at LCA, are studying how to better predict who will develop lung cancer through enhanced risk modeling, improved imaging technology, and the inclusion of biological biomarkers into the screening paradigm.
The lung cancer community has seen tremendous progress during the last several years, with screening moving from research to implementation; however, there are still opportunities to advance the field. With regard to implementation, the LCA Screening and Care Conference has become an annual event, bringing together screening implementers to learn from each other about creating successful programs and following best practices. In 2015, LCA drove the development of a bipartisan congressional Lung Cancer Caucus to educate policymakers, who will be responsible for making decisions that will continue to advance research and provide appropriate care to those at high risk for the disease. ${ }^{910}$ The National Academies of Sciences hosted a lung cancer screening workshop in 2016, which identified challenges as well as opportunities in implementation and research. ${ }^{11}$ As the field continues to advance in 2017, there is already movement underway for the creation of inter-organizational collaborative efforts that will further promote growth of this preventive service for years to come.

The LCA experience is an example of how an agile and responsive patient advocacy charity can provide critical institutional support and a national public health blueprint for the scale-up of a new preventive service that is patient focused and patient driven. Together, the lung cancer community will create a world where lung cancer is no longer considered a deadly disease, and LCA is honored to be a catalyst for that community. $\square$
1. Howlader N, Noone AM, Krapcho M, et al. (eds), SEER Cancer Statistics Review, 1975-2013, Bethesda, MD: National Cancer Institute, 2016.

2. The National Lung Screening Trial Research Team, Reduced lung-cancer mortality with low-dose computed tomographic screening, N Engl J Med, 2011;365:395-409.

3. National framework for lung screening excellence, 2012. Available at: www.lungcanceralliance.org/am-i-at-risk/national-frameworkfor-lung-screening-excellence.html (accessed March 6, 2017).

4. Centers for Medicare \& Medicaid Services, Decision memo for screening for lung cancer with low dose computed tomography (LDCT) (CAG-00439N), 2015. Available at: www.cms.gov/medicare-
coverage-database/details/nca-decision-memo.aspx?NCAld=274 (accessed March 6, 2017)

5. McKee BJ, Regis SM, McKee AB, et al., Performance of ACR LungRADS in a clinical CT lung screening program, J Am Coll Radiol, 2016;13(Suppl 2):R25-9.

6. Pinsky PF, Gierada DS, Black W, et al., Performance of Lung-RADS in the National Lung Screening Trial: a retrospective assessment, in the National Lung Screening Tria:

7. Yip R, Yankelevitz DF, Hu M, et al., Lung cancer deaths in the National Lung Screening Trial attributed to nonsolid nodules, Radiology, 2016;281:589-96.

8. Ostroff JS, Copeland A, Borderud SP, et al., Readiness of lung cancer screening sites to deliver smoking cessation treatment: current practices, oganizational priority, and perceived barriers, 2016;18:1067-75.

9. Reps Nolan, LoBiondo and Capps establish lung cancer caucus, 2015. Available at: https://nolan.house.gov/media-center/pressreleases/reps-nolan-lobiondo-and-capps-establish-lung-cancecaucus (accessed March 6, 2017)

10. Congressional lung cancer caucus. Available at: http://www. lungcanceralliance.org/lung-cancer-advocacy/lung-cancercaucus/ (accessed March 6, 2017).

11. Balogh E, Patlak M, Nass, SJ, Implementation of lung cancer screening: proceedings of a workshop, Washington, DC: The National Academies Press, 2016. 